\title{
Gauss-Kronrod Integration Rules for Cauchy Principal Value Integrals
}

\author{
By Philip Rabinowitz
}

\begin{abstract}
Kronrod extensions to two classes of Gauss and Lobatto integration rules for the evaluation of Cauchy principal value integrals are derived. Since in one frequently occurring case. the Kronrod extension involves evaluating the derivative of the integrand. a new extension is introduced using $n+2$ points which requires only values of the integrand. However, this new rule does not exist for all $n$. and when it does, several significant figures are lost in its use.
\end{abstract}

1. Introduction. In this paper we shall consider Kronrod extensions (KE) to integration rules based on Gauss and Lobatto points for the evaluation of Cauchy principal value (CPV) integrals of the form

$$
I(f ; \lambda)=f_{-1}^{1} w(x) \frac{f(x)}{x-\lambda} d x, \quad-1<\lambda<1 .
$$

Since the existence theory of KE's for regular integrals insures such extensions for only certain classes of weight functions, $w(x)$, we shall restrict our attention here to the most important class, namely $w(x)=\left(1-x^{2}\right)^{\mu-1 / 2}$, where $0 \leqslant \mu \leqslant 2$ in the Gauss case and $-\frac{1}{2}<\mu \leqslant 1$ in the Lobatto case [9]. (The only other relevant weight functions are the Jacobi weight functions $w(x)=(1-x)^{\alpha}(1+x)^{\beta}$ with $\alpha=\frac{1}{2}$, $-\frac{1}{2} \leqslant \beta \leqslant \frac{3}{2}$ or $\beta=\frac{1}{2},-\frac{1}{2} \leqslant \alpha \leqslant \frac{3}{2}$ and $w(x)=\sqrt{1-x^{2}} /\left(1-r x^{2}\right),-\infty<r \leqslant 1$ [5].) For our case, $w(x)=\left(1-x^{2}\right)^{\mu-1 / 2}$, the corresponding orthogonal polynomials are the Gegenbauer polynomials $C_{n \mu}(x)$ (usually written $C_{n}^{\mu}(x)$ ) which have the following normalization [11, p. 174]

$$
\int_{-1}^{1} w(x) C_{n \mu}(x) C_{m \mu}(x) d x=\delta_{n m} h_{n \mu},
$$

where

$$
h_{n \mu}=\sqrt{\pi} \Gamma(n+2 \mu) \Gamma\left(\mu+\frac{1}{2}\right) /(n+\mu) n ! \Gamma(\mu) \Gamma(2 \mu),
$$

which implies that $C_{n \mu}=k_{n \mu} x^{n}+\cdots$, where

$$
k_{n \mu}=2^{n} \Gamma(n+\mu) / n ! \Gamma(\mu) \text {. }
$$

$C_{n \mu}(x)$ is even (odd) if $n$ is even (odd). Special cases of $C_{n \mu}(x)$, perhaps with a different normalization, are $T_{n}(x)$, the Chebyshev polynomials of the first kind

Received October 6, 1981.

1980 Mathematics Subject Classification. Primary 65D30.

Key words and phrases. Cauchy principal value integral, Kronrod rule, Gauss integration rule, Lobatto integration rule, Gegenbauer polynomials, Szegö polynomials. 
$(\mu=0), P_{n}(x)$, the Legendre polynomials $\left(\mu=\frac{1}{2}\right)$, and $U_{n}(x)$, the Chebyshev polynomials of the second kind $(\mu=1)$.

The $C_{n \mu}(x)$ satisfy the following differential equation

$$
\left(1-x^{2}\right) C_{n \mu}^{\prime \prime}(x)-(2 \mu+1) x C_{n \mu}^{\prime}(x)+n(n+2 \mu) C_{n \mu}(x)=0,
$$

and the recurrence relation

$$
\begin{array}{r}
(n+1) C_{n+1, \mu}(x)=2 \cdot(n+\mu) x C_{n \mu}(x)-(n+2 \mu-1) C_{n-1, \mu}(x), \\
n=0,1, \ldots,
\end{array}
$$

with $C_{0 \mu}(x) \equiv 1, C_{-1, \mu}(x) \equiv 0$.

We shall now consider integration rules approximating $I(f ; \lambda)$ as well as rules approximating If $\equiv \int_{-1}^{1} w(x) f(x) d x$, since the latter are intimately connected with the former as we shall see. We shall say that an integration rule is of exact precision $N(E P=N)$ if it is exact for all polynomials of degree $\leqslant N$ and if there exists a polynomial of degree $N+1$ for which the integration rule is not exact. While in many cases, more exact information about the integration error exists, the value of the $E P$ is sufficient for our purpose.

The $n$-point Gauss-Gegenbauer integration rule (GGIR) approximating If is given by

$$
G G_{n} f=\sum_{i=1}^{n} w_{i} f\left(x_{i}\right), \quad E P=2 n-1,
$$

where we have omitted the dependence of $w_{i}$ and $x_{i}$ on $\mu$ and $n$. The abscissas $x_{i}$ are the zeros of $C_{n \mu}(x)$, and the weights $w_{i}$, as well as the weights in the next three integration rules, are interpolatory weights to be discussed below. The LobattoGegenbauer integration rule (LGIR) with $E P=2 n-1$ has $n+1$ points and is given by

$$
L G_{n+1} f=\sum_{i=1}^{n+1} \bar{w}_{i} f\left(\bar{x}_{i}\right),
$$

where the $\bar{x}_{i}$ are the zeros of $\bar{C}_{n+1, \mu+1} \equiv\left(1-x^{2}\right) C_{n-1, \mu+1}$. The KEGGIR is given by

$$
K G G_{n} f=\sum_{i=1}^{n} u_{i} f\left(x_{i}\right)+\sum_{i=1}^{n+1} v_{i} f\left(y_{i}\right),
$$

with

$$
E P= \begin{cases}3 n+1, & n \text { even }, \quad 0<\mu \leqslant 2, \mu \neq 1 \\ 3 n+2, & n \text { odd }, \\ 4 n-1, & \mu=0 \\ 4 n+1, & \mu=1\end{cases}
$$

The $y_{i}$ are the zeros of the Szegö polynomial $E_{n+1, \mu}(x)$, which we shall treat in Section 2. For the moment we state a result of Szegö [10] that, for $0 \leqslant \mu \leqslant 2$, the $y_{i}$ are real, lie in $[-1,1]$, and are separated by the $x_{i}$. (For $\mu \neq 0$, the $y_{i}$ lie in $(-1,1)$.) The corresponding KELGIR is given by

$$
K L G_{n+1} f=\sum_{i=1}^{n+1} \bar{u}_{i} f\left(\bar{x}_{i}\right)+\sum_{i=1}^{n} \bar{v}_{i} f\left(\bar{y}_{i}\right),
$$


where the $\bar{y}_{i}$ are the zeros of $E_{n, \mu+1}(x)$, and

$$
E P= \begin{cases}3 n, & n \text { odd }, \\ 3 n+1, & n \text { even }, \\ 4 n-1, & \mu=0\end{cases}
$$

As mentioned above, the weights in rules (7)-(10) are interpolatory weights defined as follows: Consider the not necessarily monic polynomial

$$
P_{N}(x)=\gamma_{N} \prod_{i=1}^{1}\left(x-z_{i}\right)
$$

where the $z_{i}$ are the integration points of a particular rule and $\gamma_{N}$ is the leading coefficient of $P_{N}(x)$. The weight $a_{i}$ corresponding to the point $z_{i}$ is given by

$$
a_{i}=\int_{-1}^{1} \frac{w(x) P_{N}(x)}{\left(x-z_{i}\right) P_{N}^{\prime}\left(z_{i}\right)} d x .
$$

This can also be written as $a_{i}=Q_{N}\left(z_{i}\right) / P_{N}^{\prime}\left(z_{i}\right)$, where the 'function of the second kind' $Q_{N}(t)$ is defined by

$$
Q_{N}(t)=I\left(P_{N} ; t\right) \equiv f_{-1}^{1} \frac{w(x) P_{N}(x)}{x-t} d x .
$$

The error $E_{N} f$ in the interpolatory integration rule may be written as

$$
E_{N} f=I f-\sum_{i=1}^{N} a_{i} f\left(z_{i}\right)=\gamma_{N}^{-1} \int_{-1}^{1} w(x) P_{N}(x) f\left[z_{1}, \ldots, z_{N}, x\right] d x,
$$

where $f\left[z_{1}, \ldots, z_{N}, x\right]$ is the $N$ th divided difference of $f(x)$ based on the points $z_{1}, \ldots, z_{N}$ and $x$. The $E P$ of such a rule depends on the nature of $P_{N}(x)$. However, if $I\left(P_{N}\right) \neq 0$, we have that $E P=N-1$.

If we wish to compute the CPV integral (1), we can follow an interpolatory path and get immediately

$$
\begin{aligned}
I(f ; \lambda) & =\sum_{i=1}^{N} b_{i} f\left(z_{i}\right)+\gamma_{N}^{-1} f_{-1}^{1} \frac{w(x) P_{N}(x) f\left[z_{1}, \ldots, z_{N}, x\right]}{x-\lambda} d x \\
& =R_{N}(f ; \lambda)+E_{N}(f ; \lambda),
\end{aligned}
$$

where

$$
b_{i}=f_{-1}^{1} \frac{w(x) P_{N}(x)}{\left(x-z_{i}\right)(x-\lambda) P_{N}^{\prime}\left(z_{i}\right)} d x, \quad i=1, \ldots, N .
$$

If $\lambda \neq z_{i}$, we can write

$$
b_{i}=\frac{a_{i}}{z_{i}-\lambda}-\frac{1}{\left(z_{i}-\lambda\right) P_{N}^{\prime}\left(z_{i}\right)} f_{-1}^{1} \frac{w(x) P_{N}(x)}{x-\lambda} d x=\frac{Q_{N}\left(z_{i}\right)-Q_{N}(\lambda)}{\left(z_{i}-\lambda\right) P_{N}^{\prime}\left(z_{i}\right)}
$$

while for $\lambda=z_{i}$ we have by limiting process that

$$
b_{i}=Q_{N}^{\prime}\left(z_{i}\right) / P_{N}^{\prime}\left(z_{i}\right)=Q_{N}^{\prime}(\lambda) / P_{N}^{\prime}(\lambda) .
$$

As above, $E P=N-1$ unless $Q_{N}(\lambda)=0$ which will occur only for a finite number of values of $\lambda$. Thus, in this approach there is no advantage to Gauss points over any other set of integration points for arbitrary $\lambda$ since, for any set of $N$ distinct points, 
$E P=N-1$. However, for those $\lambda$ such that $Q_{N}(\lambda)=0$, Gauss rules will be superior as we shall see.

To improve on this situation, we define a second class of interpolatory rules for CPV integrals, where we interpolate at the point $x=\lambda$ in addition to the points $z_{1}, \ldots, z_{N}$. If we define $\hat{P}_{N+1}(x)=(x-\lambda) P_{N}(x)$ and $z_{0}=\lambda$ assumed distinct from $z_{1}, \ldots, z_{N}$, we have

$$
\begin{aligned}
I(f ; \lambda) & =\sum_{i=0}^{N} d_{i} f\left(z_{i}\right)+\gamma_{i}^{-1} \int_{-1}^{1} w(x) P_{N}(x) f\left[z_{0}, \ldots, z_{N}, x\right] d x \\
& =\hat{R}_{N+1}(f ; \lambda)+\hat{E}_{N+1}(f ; \lambda),
\end{aligned}
$$

where

$$
d_{i}=f_{-1}^{1} \frac{w(x) \hat{P}_{N+1}(x) d x}{\left(x-z_{i}\right)\left(z_{i}-\lambda\right) \hat{P}_{N+1}^{\prime}\left(z_{i}\right)}, \quad i=0, \ldots, N
$$

For $i=1, \ldots, N$, this reduces to

$$
d_{i}=\frac{Q_{N}\left(z_{i}\right)}{\left(z_{i}-\lambda\right) P_{N}^{\prime}\left(z_{i}\right)}=\frac{a_{i}}{z_{i}-\lambda},
$$

while for $i=0$

$$
d_{0}=Q_{N}(\lambda) / P_{N}(\lambda)
$$

By comparing $\hat{E}_{N+1}(f ; \lambda)$ with $E_{N} f$ in (14), we conclude that the $E P$ of (19) is $1+E P$ of (14).

If $\lambda=z_{j}$, for some $j$, we can again apply a limiting process to get the following rule, which turns out to have a different structure from (19) in that it involves a derivative. Thus, writing

$$
\hat{R}_{N+1}(f ; \lambda)=d_{0} f(\lambda)+\sum_{\substack{i=1 \\ i \neq j}}^{N} d_{i} f\left(z_{i}\right)+d_{j} f\left(z_{j}\right)
$$

and letting $\lambda \rightarrow z_{j}$, we have

$$
\hat{R}_{N+1}(f ; \lambda)=\sum_{\substack{i=1 \\ i \neq j}}^{N} d_{i} f\left(z_{i}\right)+\lim _{\lambda \rightarrow z_{1}}\left[d_{0} f(\lambda)+d_{j} f\left(z_{j}\right)\right] .
$$

The term in brackets can be written as

$$
\begin{aligned}
& \frac{1}{z_{j}-\lambda}\left[\frac{Q_{N}\left(z_{j}\right)}{P_{N}^{\prime}\left(z_{j}\right)} f\left(z_{j}\right)-\frac{Q_{N}(\lambda)}{\prod_{i=1: i \neq j}^{N}\left(\lambda-z_{i}\right)} f(\lambda)\right] \\
& =\frac{1}{z_{j}-\lambda}\left[\frac{Q_{N}\left(z_{j}\right)}{P_{N}^{\prime}\left(z_{j}\right)}\left(f\left(z_{j}\right)-f(\lambda)\right)-\left(\frac{Q_{N}(\lambda)}{\prod_{i=1: i \neq j}^{N}\left(\lambda-z_{i}\right)}-\frac{Q_{N}\left(z_{j}\right)}{P_{N}^{\prime}\left(z_{j}\right)}\right) f(\lambda)\right] .
\end{aligned}
$$

Hence,

$$
\lim _{\lambda \rightarrow z_{j}}\left[d_{0} f(\lambda)+d_{j} f\left(z_{j}\right)\right]=a_{j} f^{\prime}\left(z_{j}\right)+\hat{a}_{j} f\left(z_{j}\right),
$$


where

$$
\hat{a}_{j}=\frac{Q_{N}\left(z_{j}\right) P_{N}^{\prime \prime}\left(z_{j}\right)}{2\left[P_{N}^{\prime}\left(z_{j}\right)\right]^{2}}-\frac{Q_{N}^{\prime}\left(z_{j}\right)}{P_{N}^{\prime}\left(z_{j}\right)}=\frac{a_{j} P_{N}^{\prime \prime}\left(z_{j}\right) / 2-Q_{N}^{\prime}\left(z_{j}\right)}{P_{N}^{\prime}\left(z_{j}\right)},
$$

so that in this case

$$
\hat{R}_{N+1}\left(f ; z_{j}\right)=\sum_{\substack{i=1 \\ i \neq j}}^{N} a_{i} \frac{f\left(z_{i}\right)}{z_{i}-\lambda}+\hat{a}_{j} f\left(z_{j}\right)+a_{j} f^{\prime}\left(z_{j}\right) .
$$

The formulas for $R_{N}(f ; \lambda)$ and $\hat{R}_{N+1}(f ; \lambda)$ derived above are not new. See, for example, Section 3.2 in the survey paper by Gautschi [3] and the references cited therein. (Note that Gautschi's formulas refer to $f(f(x) /(\lambda-x)) d x=-I(f ; \lambda)$.)

In Section 3, we shall recover the known Gauss and Lobatto type rules for CPV integrals by specializing $P_{N}(x)$ to the appropriate set of points. In addition we shall derive KE's of these rules. Now, in contrast to the application of KE's to usual GGIR's and LGIR's, it may turn out that in the CPV case the rule $\hat{R}_{N+1}(f ; \lambda)$ based on Gauss or Lobatto points does not involve a derivative, whereas the corresponding $\mathrm{KE}$ does, so that the $\mathrm{KE}$ has a different structure. This is not a rare situation! In fact, it will invariably occur when we approximate $I(f ; 0)$ by a Gauss or Lobatto rule using an even number of points, which implies that the rule is of the form (19), whereas the KE which has $x=0$ as one of the integration points will be of the form (28). Furthermore, for $w(x) \equiv 1$, the most important case, any CPV integral $I(f ; \lambda)$ can be reduced to the sum of a regular integral and $I(g ; 0)$ for some $g$ closely related to $f$. Since it is not always convenient to evaluate derivatives, we investigate in Section 4 another possibility for extending $2 m$-point Gauss and Lobatto rules by adding $2 m+2$ points rather than $2 m+1$ points as in the Kronrod case, thus avoiding the point $x=0$. We shall define a polynomial $\tilde{E}_{2 m+2, \mu}(z)$ closely related to the Szegö polynomial $E_{2 m+1, \mu}(z)$, whose zeros are the required integration points. However, it turns out that $\tilde{E}_{2 m+2, \mu}(x)$ does not always have $2 m+2$ real zeros, and when it does, the ensuing integration rule loses a few significant figures, the number increasing with $n$. As a byproduct of our investigation we get a partial answer to the question of the existence of KE's for Gauss rules with respect to the Jacobi weight functions $w(x)=(1-x)^{\alpha}(1+x)^{\beta}$ with $\alpha=-\frac{1}{2},-\frac{1}{2}<\beta \leqslant \frac{3}{2}, \beta \neq \frac{1}{2}$ and $\beta=-\frac{1}{2},-\frac{1}{2}<\alpha \leqslant \frac{3}{2}, \alpha \neq \frac{1}{2}$.

While this paper is mostly of a theoretical nature, we believe that the results presented have practical implications for the computation of CPV integrals. The use of Gauss-Kronrod pairs in numerical integration is well established by now [3, Section 2.1.2], [5], so that the extension to CPV integrals is only natural. There is a considerable literature on numerical evaluation of CPV integrals; see, for example, the survey [8]. Automatic programs have been published by Piessens et al. [7] and in QUADPACK [6], the latter program also appearing in the NAG library. However, these programs are for a single CPV integral. The Gauss-Kronrod approach is much more efficient when we wish to evaluate $I(f ; \lambda)$ for a series of values of $\lambda$ in $(-1,1)$. Then, provided we choose a Gauss-Kronrod pair with abscissas not too close to the $\lambda$ of interest, we have a useful tool for computing CPV integrals together with error estimates. 
If $\lambda$ is close to one of the integration points, the formulas for $R_{N}(f ; \lambda)$ and $\hat{R}_{N+1}(f ; \lambda)$ are numerically unstable. For a stable way to evaluate $R_{N}(f ; \lambda)$ in the Gauss case, see [3, Section 3.2.3]. Unfortunately, this process does not carry over to the Kronrod extension, so that to preserve accuracy in this case, one must use higher precision arithmetic. For $\hat{R}_{N+1}(f ; \lambda)$, Elliott and Paget [2] give an algorithm similar to that for $R_{N}(f ; \lambda)$ for use in the Gauss case. A close inspection of this algorithm reveals that it requires the computation of $\left(f\left(z_{i}\right)-f(\lambda)\right) /\left(z_{i}-\lambda\right)$ for all abscissas $z_{i}$. If this term can be computed accurately for all $z_{i}$, then the rule $\hat{R}_{N+1}(f ; \lambda)$ can be rewritten in such a way as to require computation of a similar term so that the Kronrod extension is no more unstable than the Gauss rule. Thus, if $\lambda$ is close to $z_{j}$ for some $j$, then, referring to (23)-(25), we have that

$$
\hat{R}_{N+1}(f ; \lambda)=\sum_{\substack{i=1 \\ i \neq j}}^{N} d_{i} f\left(z_{i}\right)+\frac{1}{z_{j}-\lambda}\left[\frac{Q_{N}\left(z_{j}\right)}{P_{N}^{\prime}\left(z_{j}\right)} f\left(z_{j}\right)-\frac{Q_{N}(\lambda)}{\prod_{i=1 ; i \neq j}^{N}\left(\lambda-z_{i}\right)} f(\lambda)\right] .
$$

The computation of the last term requires the same care as that of

$$
\left(f\left(x_{j}\right)-f(\lambda)\right) /\left(x_{j}-\lambda\right) \text {. }
$$

2. The Szegö Polynomials $E_{n+1, \mu}$. In this section, we recall from [9] the properties of the polynomials $E_{n+1, \mu}$, which we shall need to give explicit expressions for the weights of the KE's of the Gauss and Lobatto based integration rules for C.PV integrals. We shall also need some of these properties to study the related polynomial $\tilde{E}_{2 m+2, \mu}$, which we shall introduce in Section 4 .

We have the following representations:

$$
E_{n+1, \mu}(x)=\lambda_{n \mu} \sum_{i=0}^{m} \alpha_{i \mu} T_{n+1-2 i}(x)=\lambda_{n \mu} 2^{n} \prod_{j=1}^{n+1}\left(x-y_{j}\right)
$$

where

$$
\lambda_{n \mu}=2 \Gamma(n+\mu+1) / \sqrt{\pi} \Gamma(n+2 \mu),
$$

$m=[(n+1) / 2]$, the prime indicates that the constant term, if it appears, is to be halved and the $\alpha_{i \mu}$ are the first $m+1$ coefficients in the series

$$
\sum_{i=0}^{\infty} \alpha_{i \mu} u^{i}=\left\{\sum_{j=0}^{\infty} f_{j \mu} u^{j}\right\}^{-1}
$$

with $f_{0 \mu}=1$ and

$$
f_{j \mu}=(1-\mu / j)(1-\mu /(n+\mu+j)) f_{j-1, \mu} .
$$

The $\alpha_{i \mu}$ may be computed from the triangular system $\alpha_{0 \mu}=1, \alpha_{1 \mu}=-f_{1 \mu}$, and

$$
\alpha_{k \mu}=-f_{k \mu}-\sum_{i=1}^{k-1} f_{i \mu} \alpha_{k-i, \mu}, \quad k=2, \ldots, m
$$

We have not shown the dependence of the $\alpha_{i \mu}$ on $n$. 
The connection between $E_{n+1, \mu}$ and the Gegenbauer polynomials is given by the identity

$$
C_{n \mu}(x) E_{n+1, \mu}(x)=\sum_{i=0}^{n} c_{i} C_{n+1+i, \mu}(x),
$$

from which it follows that

$$
\int_{-1}^{1} w(x) C_{n \mu}(x) E_{n+1, \mu}(x) x^{k} d x=0, \quad k=0,1, \ldots, n,
$$

so that, by the theorem in [1, p. 77], an interpolatory integration rule based on the zeros of $C_{n \mu}(x)$ and $E_{n+1, \mu}(x)$ is exact for all polynomials of degree $\leqslant 3 n+1$ which forms the basis for KEGGIR's. The $c_{k}=c_{k}(\mu, n)$ are given by

$$
c_{k}=I f_{k} / h_{n+1+k, \mu} \text {, }
$$

where

$$
f_{k}(x)=C_{n \mu}(x) E_{n+1, \mu}(x) C_{n+1+k, \mu}(x), \quad k=0,1, \ldots, n,
$$

and $h_{n+1+k, \mu}$ is given by (3).

If we now define the function of the second kind $Q_{n \mu}(t)$ by

$$
Q_{n \mu}(t)=I\left(C_{n \mu} ; t\right)=f_{-1}^{1} \frac{w(x) C_{n \mu}(x)}{x-t} d x, \quad-1<t<1
$$

which differs by a constant from the function $\tilde{Q}_{n}^{\mu}(t)$ in [9], we have that

$$
Q_{n \mu}(x) E_{n+1, \mu}(x)=\delta_{\mu}+\sum_{i=0}^{n} c_{i} Q_{n+1+i, \mu}(x),
$$

where

$$
\delta_{\mu}=2 \Gamma\left(\mu+\frac{1}{2}\right) / \Gamma(2 \mu) .
$$

As is well known, the $Q_{n \mu}(t)$ also satisfy the recurrence relation (6) with $Q_{0 \mu}(t)=$ $I(1 ; t)$ and $Q_{1 \mu}(t)=2 \mu\left[t Q_{0 \mu}(t)+h_{0 \mu}\right]$. Furthermore $Q_{n \mu} \in C^{\prime}(-1,1)$.

3. The Weights in the Integration Rules for CPV Integrals. By identifying $P_{N}(x)$ with the appropriate polynomial, we get explicit expressions for the weights $b_{i}$ and $d_{i}$ in the rules $R_{N}(f ; \lambda)$ and $\hat{R}_{N+1}(f ; \lambda)$ as well as information about the exact precision of these rules. Thus, if we set $P_{N}(x)=C_{n \mu}(x)$, the first Gauss-based rule becomes, for $\lambda \neq x_{i}$,

$$
\begin{aligned}
I(f ; \lambda)= & \sum_{i=1}^{n}\left(w_{i}-\frac{Q_{n \mu}(\lambda)}{C_{n \mu}^{\prime}\left(x_{i}\right)}\right) \frac{f\left(x_{i}\right)}{x_{i}-\lambda} \\
& +k_{n \mu}^{-1} f_{-1}^{1} \frac{w(x) C_{n \mu}(x) f\left[x_{1}, \ldots, x_{n}, x\right]}{x-\lambda} d x,
\end{aligned}
$$

where the $w_{i}$ and $x_{i}$ are as in (7). If $\lambda=x_{j}$,

$$
R_{n}\left(f ; x_{j}\right)=\sum_{\substack{i=1 \\ i \neq j}}^{n}\left(w_{i}-\frac{Q_{n \mu}\left(x_{j}\right)}{C_{n \mu}^{\prime}\left(x_{i}\right)}\right) \frac{f\left(x_{i}\right)}{x_{i}-x_{j}}+\frac{Q_{n \mu}^{\prime}\left(x_{j}\right)}{C_{n \mu}^{\prime}\left(x_{j}\right)} f\left(x_{j}\right) .
$$


We get the KE of this rule by setting $P_{v}(x)=C_{n \mu}(x) E_{n+1, \mu}(x)$. Then, for $\lambda \neq x_{i}, y_{i}$

$$
\begin{aligned}
I(f ; \lambda)= & \sum_{i=1}^{n}\left(u_{i}-u_{i}^{*}\right) \frac{f\left(x_{i}\right)}{x_{i}-\lambda}+\sum_{i=1}^{n+1}\left(v_{i}-v_{i}^{*}\right) \frac{f\left(y_{i}\right)}{y_{i}-\lambda} \\
+ & +k_{n \mu}^{-1} 2^{-n} \lambda_{n \mu}^{-1} f_{-1}^{1} \frac{w(x) C_{n \mu}(x) E_{n+1, \mu}(x)}{x-\lambda} \\
& \quad \times f\left[x_{1}, \ldots, x_{n}, y_{1}, \ldots, y_{n+1}, x\right] d x,
\end{aligned}
$$

where the $x_{i}, y_{i}, u_{i}$ and $v_{i}$ are as in (9),

$$
u_{i}^{*}=\left(Q_{n \mu}(\lambda) E_{n+1, \mu}(\lambda)-\delta_{\mu}\right) / E_{n+1, \mu}\left(x_{1}\right) C_{n \mu}^{\prime}\left(x_{i}\right), \quad i=1, \ldots, n,
$$

and

$$
v_{i}^{*}=\left(Q_{n \mu}(\lambda) E_{n+1, \mu}(\lambda)-\delta_{\mu}\right) / E_{n+1, \mu}^{\prime}\left(y_{i}\right) C_{n \mu}\left(y_{i}\right), \quad i=1, \ldots, n+1 .
$$

The value of the numerators in (44) and (45) is derived as follows, using (34), (38), and (39):

$$
\begin{aligned}
Q_{N}(\lambda) & =f_{-1}^{1} w(x) \frac{C_{n \mu}(x) E_{n+1, \mu}(x)}{x-\lambda} d x \\
& =\sum_{i=0}^{n} c_{i} f_{-1}^{1} \frac{w(x) C_{n+1+i, \mu}(x)}{x-\lambda} d x \\
& =\sum_{i=0}^{n} c_{i} Q_{n+1+i, \mu}(\lambda)=Q_{n \mu}(\lambda) E_{n+1, \mu}(\lambda)-\delta_{\mu} .
\end{aligned}
$$

As for the denominators, the first or second of the two terms in the sum

$$
\left(E_{n+1, \mu}(x) C_{n \mu}(x)\right)^{\prime}=E_{n+1, \mu}^{\prime}(x) C_{n \mu}(x)+E_{n+1, \mu}(x) C_{n \mu}^{\prime}(x)
$$

vanishes for $x=x_{i}$ or $x=y_{i}$, respectively.

If $\lambda=x_{j}$, the coefficient of $f\left(x_{j}\right)$ in (43) is replaced by

$$
\frac{Q_{n \mu}^{\prime}\left(x_{j}\right)}{C_{n \mu}^{\prime}\left(x_{j}\right)}+\frac{Q_{n \mu}\left(x_{j}\right) E_{n+1, \mu}^{\prime}\left(x_{j}\right)}{C_{n \mu}^{\prime}\left(x_{j}\right) E_{n+1, \mu}\left(x_{j}\right)},
$$

while if $\lambda=y_{j}$, the coefficient of $f\left(y_{j}\right)$ in (43) is replaced by $Q_{n \mu}\left(y_{j}\right) / C_{n \mu}\left(y_{j}\right)$. This follows from (18) by differentiating (46). If we compare the Gauss-based rule (41) with its KE (43) for the important case $\lambda=0$ and $n$ even $=2 m$, we see that since $Q_{2 m, \mu}(0)=0$, the rule in (41) reduces to $G G_{n}(f / x)$, ignoring the fact that we are dealing with a CPV integral. In the corresponding $\mathrm{KE}$, we are not so fortunate inasmuch as $u_{i}^{*}$ and $v_{i}^{*}$ do not vanish for $\lambda=0$ because of the $\delta_{\mu}$ component. However, since $y_{j}=0$ for some $j$ in the KE of an even-point rule, we see that the coefficient of $f(0)$ vanishes so that the KE contains only $2 n$ rather than $2 n+1$ points. As for the error, in (41), as we shall see, $E P=2 n$ and this is also the $E P$ of the KE so that nothing is gained in this case. Of course, for arbitrary $\lambda$, when $Q_{n}(\lambda) \neq 0$, the KE increases the $E P$ from $n-1$ to $2 n$, but as we have indicated above, this could have been achieved by any $(n+1)$-point extension to any $n$-point interpolatory rule. 
In the Lobatto case, we set $P_{N}(x)=\bar{C}_{n+1, \mu+1}(x)=\left(1-x^{2}\right) C_{n-1, \mu+1}(x)$ and get a similar set of formulas. Thus, for $\lambda \neq \bar{x}_{i}$,

$$
\begin{aligned}
I(f ; \lambda)= & \sum_{i=1}^{n+1}\left(\bar{w}_{i}-\frac{Q_{n-1, \mu+1}(\lambda)}{\bar{C}_{n+1, \mu+1}^{\prime}\left(\bar{x}_{i}\right)}\right) \frac{f\left(\bar{x}_{i}\right)}{\bar{x}_{i}-\lambda} \\
& +k_{n-1, \mu+1}^{-1} f_{-1}^{1} \frac{w(x) \bar{C}_{n+1, \mu+1}(x)}{x-\lambda} f\left[\bar{x}_{1}, \ldots, \bar{x}_{n+1}, x\right] d x,
\end{aligned}
$$

where the $\bar{w}_{i}$ and $\bar{x}_{i}$ are as in (8). In the case $\lambda=\bar{x}_{j}$ for some $j$ such that $C_{n-1, \mu+1}\left(\bar{x}_{j}\right)=0(\lambda \neq \pm 1$ since $I(f ; \lambda)$ is only defined for $\lambda \in(-1,1))$, the coefficient of $f\left(\bar{x}_{j}\right)$ is replaced by $Q_{n-1, \mu+1}^{\prime}\left(\bar{x}_{j}\right) /\left(1-\bar{x}_{j}^{2}\right) C_{n-1, \mu+1}^{\prime}\left(\bar{x}_{j}\right)$. For the KE of this rule, $P_{N}(x)=\bar{C}_{n+1, \mu+1}(x) E_{n, \mu+1}(x)$, and we have for $\lambda \neq \bar{x}_{i}, \bar{y}_{i}$

$$
\begin{aligned}
I(f ; \lambda)= & \sum_{i=1}^{n+1}\left(\bar{u}_{i}-\bar{u}_{i}^{*}\right) \frac{f\left(\bar{x}_{i}\right)}{\bar{x}_{i}-\lambda}+\sum_{i=1}^{n}\left(\bar{v}_{i}-\bar{v}_{i}^{*}\right) \frac{f\left(\bar{y}_{i}\right)}{\bar{y}_{i}-\lambda} \\
+k_{n-1, \mu+1}^{-1} 2^{-n+1} \lambda_{n-1, \mu+1}^{-1} f_{-1}^{1} \frac{w(x) \bar{C}_{n+1, \mu+1}(x) E_{n, \mu+1}(x)}{x-\lambda} & \times f\left[\bar{x}_{1}, \ldots, \bar{x}_{n+1}, \bar{y}_{1}, \ldots, \bar{y}_{n}, x\right] d x,
\end{aligned}
$$

where

$$
\bar{u}_{i}^{*}=\left(Q_{n-1, \mu+1}(\lambda) E_{n, \mu+1}(\lambda)-\delta_{\mu+1}\right) / E_{n, \mu+1}\left(\bar{x}_{i}\right) \bar{C}_{n+1, \mu+1}^{\prime}\left(\bar{x}_{i}\right)
$$

and

$$
\bar{v}_{i}^{*}=\left(Q_{n-1, \mu+1}(\lambda) E_{n, \mu+1}(\lambda)-\delta_{\mu+1}\right) / E_{n, \mu+1}^{\prime}\left(\bar{y}_{i}\right) \bar{C}_{n+1, \mu+1}\left(\bar{y}_{i}\right) .
$$

If $\lambda=\bar{x}_{j}$, the coefficient of $f\left(\bar{x}_{j}\right)$ in (48) is replaced by

$$
\frac{Q_{n-1, \mu+1}^{\prime}\left(\bar{x}_{j}\right)}{\bar{C}_{n+1, \mu+1}^{\prime}\left(\bar{x}_{j}\right)}+\frac{Q_{n-1, \mu+1}\left(\bar{x}_{j}\right) E_{n, \mu+1}^{\prime}\left(\bar{x}_{j}\right)}{\bar{C}_{n+1, \mu+1}^{\prime}\left(\bar{x}_{j}\right) E_{n, \mu+1}\left(\bar{x}_{j}\right)},
$$

while if $\lambda=\bar{y}_{j}$, the coefficient of $f\left(\bar{y}_{j}\right)$ in (48) is replaced by

$$
Q_{n-1, \mu+1}\left(\bar{y}_{j}\right) / \bar{C}_{n+1, \mu+1}\left(\bar{y}_{j}\right) \text {. }
$$

The same remarks as above about the error for general $\lambda$ apply here too, in that in (47) $E P=n$ and in (48) $E P=2 n$. Similarly, the special case $\lambda=0$ and $n$ odd leads to the same conclusions as in the Gauss case.

We now turn to the rule $\hat{R}_{N+1}(f ; \lambda)$ and its error term $\hat{E}_{N+1}(f ; \lambda)$. In the Gauss case, we have that for $\lambda \neq x_{i}$

$$
\begin{aligned}
I(f ; \lambda)= & \sum_{i=1}^{n} w_{i} \frac{f\left(x_{i}\right)}{x_{i}-\lambda}+\frac{Q_{n \mu}(\lambda)}{C_{n \mu}(\lambda)} f(\lambda) \\
& +k_{n \mu}^{-1} \int_{-1}^{1} w(x) C_{n \mu}(x) f\left[x_{1}, \ldots, x_{n}, \lambda, x\right] d x
\end{aligned}
$$

and $E P=2 n$ because of orthogonality. Note that, for $\lambda=0$ and $n=2 m$, the rules (41) and (51) are identical so that, for (41), $E P=2 n$ as mentioned above. If $\lambda=x_{j}$, the two terms

$$
\frac{w_{j} f\left(x_{j}\right)}{x_{j}-\lambda}+\frac{Q_{n \mu}(\lambda) f(\lambda)}{C_{n \mu}(\lambda)}
$$


must be replaced by $\hat{w}_{j} f\left(x_{j}\right)+w_{j} f^{\prime}\left(x_{j}\right)$ with

$$
\hat{w}_{j}=\frac{w_{j} C_{n \mu}^{\prime \prime}\left(x_{j}\right) / 2-Q_{n \mu}^{\prime}\left(x_{j}\right)}{C_{n \mu}^{\prime}\left(x_{j}\right)}=\frac{w_{j}\left(\mu+\frac{1}{2}\right) x_{j}}{1-x_{j}^{2}}-\frac{Q_{n \mu}^{\prime}\left(x_{j}\right)}{C_{n \mu}^{\prime}\left(x_{j}\right)},
$$

where we have used (5).

The KE of this rule has three forms depending on the value of $\lambda$. If $\lambda \neq x_{i}, y_{i}$, then

$$
\begin{aligned}
I(f ; \lambda)= & \sum_{i=1}^{n} u_{i} \frac{f\left(x_{i}\right)}{x_{i}-\lambda}+\sum_{i=1}^{n+1} v_{i} \frac{f\left(y_{i}\right)}{y_{i}-\lambda}+v_{0} f(\lambda) \\
+ & k_{n \mu}^{-1} 2^{-n} \lambda_{n \mu}^{-1} \int_{-1}^{1} w(x) C_{n \mu}(x) E_{n+1, \mu}(x) \\
& \quad \times f\left[x_{1}, \ldots, x_{n}, y_{1}, \ldots, y_{n+1}, \lambda, x\right] d x,
\end{aligned}
$$

where

$$
v_{0}=\frac{Q_{n \mu}(\lambda)}{C_{n \mu}(\lambda)}-\frac{\delta_{\mu}}{E_{n+1, \mu}(\lambda) C_{n \mu}(\lambda)}
$$

Thus, the only additional quantity we need here is the second term in (54). The EP of (53) is equal to $1+E P$ of $K G G_{n} f$.

If $\lambda=x_{j}$, then $u_{j} f\left(x_{j}\right) /\left(x_{j}-\lambda\right)+v_{0} f(\lambda)$ must be replaced by $u_{j} f^{\prime}\left(x_{j}\right)+$ $\hat{u}_{j} f\left(x_{j}\right)$, where

$$
\begin{aligned}
\hat{u}_{j}= & u_{j}\left(\frac{E_{n+1, \mu}\left(x_{j}\right)}{E_{n+1, \mu}^{\prime}\left(x_{j}\right)}+\frac{\left(\mu+\frac{1}{2}\right) x_{j}}{1-x_{j}^{2}}\right) \\
& -\frac{Q_{n \mu}\left(x_{j}\right) E_{n+1, \mu}^{\prime}\left(x_{j}\right)+Q_{n \mu}^{\prime}\left(x_{j}\right) E_{n+1, \mu}\left(x_{j}\right)}{C_{n \mu}^{\prime}\left(x_{j}\right) E_{n+1, \mu}\left(x_{j}\right)} .
\end{aligned}
$$

While this involves a more complicated coefficient for $f\left(x_{j}\right)=f(\lambda)$, it does not involve any function values aside from the $\mathrm{KE}$ values inasmuch as the Gauss formula had already included the derivative term. The situation is different if $\lambda=y_{j}$. For then, the terms $v_{j} f\left(y_{j}\right) /\left(y_{j}-\lambda\right)+v_{0} f(\lambda)$ in (53) must be replaced by $v_{j} f^{\prime}\left(y_{j}\right)$ $+\hat{v}_{j} f\left(y_{j}\right)$, where

$$
\hat{v}_{j}=\frac{v_{j} E_{n+1, \mu}^{\prime \prime}\left(y_{j}\right)}{2 E_{n+1, \mu}^{\prime}\left(y_{j}\right)}+\frac{v_{j} C_{n \mu}^{\prime}\left(y_{j}\right)-Q_{n \mu}\left(y_{j}\right)}{C_{n \mu}\left(y_{j}\right)} .
$$

Here we have an extension which involves $f^{\prime}\left(y_{j}\right)=f^{\prime}(\lambda)$, whereas the Gauss formula did not involve $f^{\prime}(\lambda)$ at all. This is a serious drawback of this extension since it occurs in the frequent case where $\lambda=0$ and we use an even Gauss rule (also called symmetric pairing) in which case the KE contains an odd number of points including one at $x=0=\lambda$. In Section 4 , we shall suggest an alternate extension which sidesteps this problem. 
Returning to $\hat{R}_{N+1}(f ; \lambda)$, we list now the formulas in the Lobatto case and the corresponding KE's. For $\lambda \neq x_{i}$, we have

$$
\begin{aligned}
I(f ; \lambda)= & \sum_{i=1}^{n+1} \bar{w}_{i} \frac{f\left(\bar{x}_{i}\right)}{\bar{x}_{i}-\lambda}+\frac{Q_{n-1, \mu+1}(\lambda)}{\left(1-\lambda^{2}\right) C_{n-1, \lambda+1}(\lambda)} f(\lambda) \\
& +k_{n-1, \mu+1}^{-1} \int_{-1}^{1} w(x)\left(1-x^{2}\right) C_{n-1, \mu+1}(x) \\
& \quad \times f\left[\bar{x}_{1}, \ldots, \bar{x}_{n+1}, \lambda, x\right] d x .
\end{aligned}
$$

As in (51) $E P=2 n$ because of orthogonality. If $\lambda=\bar{x}_{j}$ where $C_{n-1, \mu+1}\left(\bar{x}_{j}\right)=0$, the terms involving $f\left(\bar{x}_{j}\right)$ and $f(\lambda)$ must be replaced by $\bar{w}_{j} f^{\prime}\left(\bar{x}_{j}\right)+\tilde{w}_{j} f\left(\bar{x}_{j}\right)$, where

$$
\tilde{w}_{j}=\bar{w}_{j} \frac{\left(\mu-\frac{1}{2}\right) \bar{x}_{j}}{1-\bar{x}_{j}^{2}}-\frac{Q_{n-1, \mu+1}^{\prime}\left(\bar{x}_{j}\right)}{\left(1-\bar{x}_{j}^{2}\right) C_{n-1, \mu+1}^{\prime}\left(\bar{x}_{j}\right)} .
$$

As for the KE, the three forms are, first for $\lambda \neq \bar{x}_{i}, \bar{y}_{i}$,

$$
\begin{aligned}
I(f ; \lambda)= & \sum_{i=1}^{n+1} \bar{u}_{i} \frac{f\left(\bar{x}_{i}\right)}{\bar{x}_{i}-\lambda}+\sum_{i=1}^{n} \bar{v}_{i} \frac{f\left(\bar{y}_{i}\right)}{\bar{y}_{i}-\lambda}+\bar{v}_{0} f(\lambda) \\
+ & +k_{n-1, \mu+1}^{-1} 2^{-n+1} \lambda_{n-1, \mu+1}^{-1} \int_{-1}^{1} w(x)\left(1-x^{2}\right) \\
& \quad \times C_{n-1, \mu+1}(x) E_{n, \mu+1}(x) f\left[\bar{x}_{1}, \ldots, \bar{x}_{n+1}, \bar{y}_{1}, \ldots, \bar{y}_{n}, \lambda, x\right] d x
\end{aligned}
$$

where

$$
\bar{v}_{0}=\frac{Q_{n-1, \mu+1}(\lambda)}{\left(1-\lambda^{2}\right) C_{n-1, \mu+1}(\lambda)}-\frac{\delta_{\mu+1}}{\left(1-\lambda^{2}\right) C_{n-1, \mu+1}(\lambda) E_{n, \mu+1}(\lambda)} .
$$

The $E P$ of (59) is equal to $1+E P$ of $K L G_{n+1} f$.

If $\lambda=\bar{x}_{j}$ where $C_{n-1, \mu+1}\left(\bar{x}_{j}\right)=0$, the sum $\bar{u}_{j} f\left(\bar{x}_{j}\right) /\left(x_{j}-\lambda\right)+\bar{v}_{0} f(\lambda)$ must be replaced by $\bar{u}_{j} f^{\prime}\left(\bar{x}_{j}\right)+\tilde{u}_{j} f\left(\bar{x}_{j}\right)$, where

$$
\begin{aligned}
\tilde{u}_{j}= & \bar{u}_{j}\left(\frac{E_{n, \mu+1}\left(\bar{x}_{j}\right)}{E_{n, \mu+1}^{\prime}\left(\bar{x}_{j}\right)}+\frac{\left(\mu-\frac{1}{2}\right) \bar{x}_{j}}{1-\bar{x}_{j}^{2}}\right) \\
& -\frac{\left(Q_{n-1, \mu+1}\left(\bar{x}_{j}\right) E_{n, \mu+1}^{\prime}\left(\bar{x}_{j}\right)+Q_{n-1, \mu+1}^{\prime}\left(\bar{x}_{j}\right) E_{n, \mu+1}\left(\bar{x}_{j}\right)\right)}{\bar{C}_{n+1, \mu+1}^{\prime}\left(\bar{x}_{j}\right) E_{n, \mu+1}\left(\bar{x}_{j}\right)},
\end{aligned}
$$

while if $\lambda=\bar{y}_{j}$, the sum $\bar{v}_{j} f\left(\bar{y}_{j}\right) /\left(\bar{y}_{j}-\lambda\right)+\bar{v}_{0} f(\lambda)$ must be replaced by $\bar{v}_{j} f^{\prime}\left(\bar{y}_{j}\right)+$ $\tilde{v}_{j} f\left(\bar{y}_{j}\right)$, where

$$
\tilde{v}_{j}=\frac{\bar{v}_{j} E_{n, \mu+1}^{\prime \prime}\left(\bar{y}_{j}\right)}{2 \bar{E}_{n, \mu+1}^{\prime}\left(\bar{y}_{j}\right)}+\frac{\bar{v}_{j} \bar{C}_{n+1, \mu+1}^{\prime}\left(\bar{y}_{j}\right)-Q_{n-1, \mu+1}\left(\bar{y}_{j}\right)}{\left(1-\bar{y}_{j}^{2}\right) C_{n-1, \mu+1}\left(\bar{y}_{j}\right)} .
$$

The remarks above about Gauss rules for $I(f ; 0)$ with $n$ even apply here for odd $n$.

4. The Orthogonal Polynomials $\tilde{E}_{2 m+2, \mu}$. In this section we introduce and derive some properties of the polynomial of degree $n+2, n=2 m, \tilde{E}_{n+2, \mu}$, which satisfies the orthogonality conditions

$$
\int_{-1}^{1} w(x) C_{n \mu}(x) \tilde{E}_{n+2, \mu}(x) x^{k} d x=0, \quad k=0,1, \ldots, n+1 .
$$


Note that for $n$ odd, we already have that

$$
\int_{-1}^{1} w(x) C_{n \mu}(x) E_{n+1, \mu}(x) x^{k} d x=0, \quad k=0,1, \ldots, n+1,
$$

by the odd-even effect. Now, if we consider the polynomial of degree $n+2$, $x E_{n+1, \mu}(x)-a$, for any constant $a$, we have that

$$
\int_{-1}^{1} w(x) C_{n \mu}(x)\left(x E_{n+1, \mu}(x)-a\right) x^{k} d x=0, \quad k=0,1, \ldots, n-1 .
$$

by (35) and the orthogonality of $C_{n \mu}$. Hence, if we define $\tilde{E}_{n+2, \mu}(x)=x E_{n+1 . \mu}(x)-$ $\tilde{a}_{n \mu}$, where

$$
\tilde{a}_{n \mu}=\int_{-1}^{1} w(x) C_{n \mu}(x) E_{n+1, \mu}(x) x^{n+1} d x / \int_{-1}^{1} w(x) C_{n \mu}(x) x^{n} d x,
$$

it follows that (63) holds for $k=n$ and, by the odd-even effect, also for $k=n+1$. An explicit expression for $\tilde{a}_{n \mu}$ follows from some results in [9] and the properties of the orthogonal polynomials $C_{n \mu}$, so that

$$
\tilde{a}_{n \mu}=\frac{c_{0} h_{n+1, \mu}}{k_{n+1, \mu}} / \frac{h_{n \mu}}{k_{n \mu}}=\left(\alpha_{m \mu}-\alpha_{m+1, \mu}\right) \lambda_{n \mu} / 4 .
$$

Monegato [4] has shown that the polynomial $\tilde{E}_{n+2, \mu}$ defined above is the unique (up to a constant multiplier) polynomial of degree $n+2$ satisfying (63).

We now investigate the zeros of $\tilde{E}_{n+2, \mu}$. Since, for $n$ even, $\tilde{E}_{n+2, \mu}$ is an even function, it suffices to deal with the positive zeros. First we see that at the zeros $y_{i}$ of $E_{n+1, \mu}, \tilde{E}_{n+2, \mu}\left(y_{i}\right)=-\tilde{a}_{n \mu}$. Furthermore, $\tilde{E}_{n+2, \mu}^{\prime}(0)=0$, so that $\tilde{E}_{n+2, \mu}$ has a maximum or minimum at $x=0$ depending on the sign of $\tilde{E}_{n+2, \mu}^{\prime \prime}(0)$. Since $\tilde{E}_{n+2, \mu}^{\prime \prime}(0)=$ $2 E_{n+1, \mu}^{\prime}(0)$, this sign depends on the parity of $m=n / 2$. For $m$ even, $E_{n+1, \mu}^{\prime}(0)>0$, while for $m$ odd, $E_{n+1, \mu}^{\prime}(0)<0$ as can be verified by tracing the curve of the polynomial of odd degree $E_{n+1, \mu}$. Thus, for $m$ odd, $\tilde{E}_{n+2, \mu}$ has a maximum at $x=0$, while for even $m$, it has a minimum.

Let us now consider the case $0<\mu<1$. From the results in [9], we see that $\tilde{a}_{n \mu}<0$. Now, if $m$ is even, $\tilde{E}_{m+2, \mu}$ has a minimum value of $-\tilde{a}_{n \mu}>0$ at $x=0$. If we label the nonnegative zeros of $E_{n+1, \mu}$ as $0=y_{0}<y_{1}<\cdots<y_{m}<1$, then in every interval $\left(y_{i}, y_{i+1}\right), \tilde{E}_{n+2, \mu}$ has an extreme point. Hence, $\tilde{E}_{n+2, \mu}$ has a maximum in $\left(y_{0}, y_{1}\right)$, a minimum in $\left(y_{1}, y_{2}\right), \ldots$, and, finally, a minimum in $\left(y_{m-1}, y_{m}\right)$. In each interval in which $\tilde{E}_{n+2, \mu}$ has a maximum, it has no zeros. If it has a minimum in an interval, it will have no zeros, a double zero, or two zeros there depending on whether the minimum value of $\tilde{E}_{n+2, \mu}$ is positive, zero, or negative. Thus the maximum number of positive zeros is $m$, so that $\tilde{E}_{n+2, \mu}$ has at most $n$ real zeros for $m$ even and $0<\mu<1$. On the other hand, if $m$ is odd, $E_{n+2, \mu}$ may have $n+2$ real zeros and most probably does inasmuch as $\tilde{a}_{n \mu}$ is small so that $\tilde{E}_{n+2, \mu}$ is indeed negative at the minimum points. In fact, the zeros of $\tilde{E}_{n+2, \mu}$ are quite close to the zeros of $E_{n+1, \mu}$.

For $1<\mu \leqslant 2$, the situation is reversed inasmuch as $\tilde{a}_{n \mu}>0$ [9], so that in every interval in which $\tilde{E}_{n+2, \mu}$ has a minimum there are no zeros, while when $\tilde{E}_{n+2, \mu}$ has a maximum there are no zeros, a double zero, or two zeros depending on whether the 
value of $\tilde{E}_{n+2, \mu}$ at this maximum is negative, zero, or positive. Thus, for $m$ odd and $1<\mu \leqslant 2, \tilde{E}_{n+2, \mu}$ has at most $n$ real zeros, while for $m$ even, $\tilde{E}_{n+2, \mu}$ may, and most probably does, have $n+2$ real zeros which are quite close to the zeros of $E_{n+1, \mu}$.

Before discussing the use of these results in numerical integration, we remark here that Monegato [5] has shown that

$$
\tilde{E}_{2 m+2, \mu}(x)=E_{m+1}^{(\mu+1 / 2 .-1 / 2)}\left(2 x^{2}-1\right)=(-1)^{m+1} E_{m+1}^{(-1 / 2 . \mu+1 / 2)}\left(1-2 x^{2}\right)
$$

where $E_{m+1}^{(\alpha, \beta)}(x)$ satisfies the orthogonality conditions

$$
\int_{-1}^{1}(1-x)^{\alpha}(1+x)^{\beta} P_{m}^{(\alpha, \beta)}(x) E_{m+1}^{(\alpha, \beta)}(x) x^{k} d x=0, \quad k=0, \ldots, m,
$$

and $P_{m}^{(\alpha, \beta)}$ is the Jacobi polynomial. Our investigation therefore shows that a Kronrod extension to the Gauss-Jacobi integration rule based on the zeros of $P_{m}^{(\mu+1 / 2,-1 / 2)}$ or $P_{m}^{(-1 / 2, \mu+1 / 2)}$ with all zeros in $[-1,1]$ cannot exist for $m$ even and $0<\mu<1$ and for $m$ odd and $1<\mu \leqslant 2$. For $m$ odd and $0<\mu<1$ and for $m$ even and $1<\mu \leqslant 2$, KE's can probably be computed even though the theory is incomplete.

Once we have a situation where we have $n+2$ zeros $\tilde{y}_{i}$ of $\tilde{E}_{n+2, \mu}$, we can use them to derive an extension to $G G_{n} f$. We have that

$$
\begin{aligned}
I f= & \sum_{i=1}^{n} r_{i} f\left(x_{i}\right)+\sum_{i=1}^{n+2} s_{i} f\left(\tilde{y}_{i}\right) \\
& +k_{n \mu}^{-1} 2^{-n} \lambda_{n \mu}^{-1} \int_{-1}^{1} w(x) C_{n \mu}(x) \tilde{E}_{n+2, \mu}(x) f\left[x_{1}, \ldots, x_{n}, \tilde{y}_{1}, \ldots, \tilde{y}_{n+2}, x\right] d x,
\end{aligned}
$$

with $E P=3 n+3$, where

$$
\begin{gathered}
r_{i}=\frac{x_{i} u_{i} E_{n+1, \mu}\left(x_{i}\right)-\tilde{a}_{n \mu} w_{i}}{x_{i} E_{n+1, \mu}\left(x_{i}\right)-\tilde{a}_{n \mu}}, \\
s_{i}=\frac{-\delta_{\mu}}{C_{n \mu}\left(\tilde{y}_{i}\right)\left(E_{n+1, \mu}\left(\tilde{y}_{i}\right)+\tilde{y}_{i} E_{n+1, \mu}^{\prime}\left(\tilde{y}_{i}\right)\right)},
\end{gathered}
$$

and $x_{i}, u_{i}, w_{i}$ are as in (7) and (9). From (19)-(22) with $P_{N}(x)=C_{n \mu}(x) \tilde{E}_{n+2, \mu}(x)$, we have that

$$
\begin{aligned}
I(f ; \lambda)= & \sum_{i=1}^{n} r_{i} \frac{f\left(x_{i}\right)}{x_{i}-\lambda}+\sum_{i=1}^{n+2} s_{i} \frac{f\left(\tilde{y}_{i}\right)}{\tilde{y}_{i}-\lambda}+s_{0} f(\lambda) \\
+ & k_{n \mu}^{-1} 2^{-n} \lambda_{n \mu}^{-1} \int_{-1}^{1} w(x) C_{n \mu}(x) \tilde{E}_{n+2, \mu}(x) \\
& \quad \times f\left[x_{1}, \ldots, x_{n}, \tilde{y}_{1}, \ldots, \tilde{y}_{n+2}, \lambda, x\right] d x
\end{aligned}
$$

with $E P=3 n+4$, where

$$
s_{0}=\frac{Q_{n \mu}(\lambda)}{C_{n \mu}(\lambda)}-\frac{\lambda \delta_{\mu}}{\tilde{E}_{n+2, \mu}(\lambda) C_{n \mu}(\lambda)} .
$$


(71), (72), and (74) follow from the fact that

$$
\begin{aligned}
Q_{N}(t) & =f_{-1}^{1} \frac{w(x) C_{n \mu}(x) \tilde{E}_{n+2, \mu}(x)}{x-t} d x \\
& =f_{-1}^{1} \frac{w(x) C_{n \mu}(x)\left[x E_{n+1, \mu}(x)-\tilde{a}_{n \mu}\right]}{x-t} d x \\
& =t f_{-1}^{1} \frac{w(x) C_{n \mu}(x) E_{n+1, \mu}(x)}{x-t} d x-\tilde{a}_{n \mu} Q_{n \mu}(t) \\
& =t\left[Q_{n \mu}(t) E_{n+1, \mu}(t)-\delta_{\mu}\right]-\tilde{a}_{n \mu} Q_{n \mu}(t) \\
& =Q_{n \mu}(t) \tilde{E}_{n+2, \mu}(t)-t \delta_{\mu} .
\end{aligned}
$$

We are only interested in the case $\lambda \neq x_{i}, \tilde{y}_{i}$, since otherwise we would use the usual KE's given in Section 3. We notice that, as before, for $\lambda=0, s_{0}=0$ and we have a case of symmetric pairing. Unfortunately, for the very value $\lambda=0$, for which we developed this new formula, there are problems. In Table 1, we list some values of $\tilde{a}_{n \mu}$ and $\tilde{y}_{1}$, the smallest positive zero of $\tilde{E}_{n+2, \mu}$ for $\mu=\frac{1}{2}$, the most prevalent case. Since the integration rule in (73) can be rewritten, for $\lambda=0$, as

$$
\sum_{i=1}^{m} r_{i} \frac{\left[f\left(x_{i}\right)-f\left(-x_{i}\right)\right]}{x_{i}}+\sum_{i=1}^{m+1} s_{i} \frac{\left[f\left(\tilde{y}_{i}\right)-f\left(-\tilde{y}_{i}\right)\right]}{\tilde{y}_{i}}
$$

where we assume that the positive zeros are $x_{1}<x_{2}<\cdots<x_{m}$ and $\tilde{y}_{1}<\tilde{y}_{2}<$ $\cdots<\tilde{y}_{m+1}$, we see that one term in the second sum in (75) is $s_{1}\left[f\left(\tilde{y}_{1}\right)-f\left(-\tilde{y}_{1}\right)\right] / \tilde{y}_{1}$ and, as the numbers in Table 1 show, this term can lead to the loss of several significant figures, the number increasing with increasing $n$.

TABLE 1

$\tilde{a}_{n \mu}$ and $\tilde{y}_{1}$, the first positive zero of $\tilde{E}_{n+2, \mu}$, for $\mu=\frac{1}{2}$ and odd $m, n=2 m$

$\begin{array}{rcc}m & \tilde{a}_{n \mu} & \tilde{y}_{1} \\ 3 & -1.54(-2) & 4.14664(-2) \\ 5 & -5.70(-3) & 1.97603(-2) \\ 7 & -2.46(-3) & 1.20896(-2) \\ 9 & -1.81(-3) & 8.36055(-3) \\ 11 & -1.22(-3) & 6.22128(-3) \\ 13 & -8.79(-4) & 4.86127(-3) \\ 15 & -6.63(-4) & 3.93369(-3) \\ 17 & -5.18(-4) & 3.26778(-3) \\ 19 & -4.16(-4) & 2.77069(-3) \\ 21 & -3.41(-4) & 2.38801(-3) \\ 23 & -2.85(-4) & 2.08599(-3) \\ 25 & -2.42(-4) & 1.84268(-3)\end{array}$

In the Lobatto case, the appropriate orthogonal polynomial for $n$ odd, i.e. an even number of integration points, is $\tilde{E}_{n+1, \mu+1}(x)$. In this case, we see that if $-\frac{1}{2}<\mu \leqslant 0$, 
$n=2 m+1, \tilde{E}_{n+1, \mu+1}$ has at most $2 m$ real roots if $m$ is even and probably has $2 m+2$ real roots if $m$ is odd, while for $0<\mu \leqslant 1$, which includes the usual Lobatto case, $\mu=\frac{1}{2}, \tilde{E}_{n+1, \mu+1}$ has at most $2 m$ real roots if $m$ is odd and probably has $2 m+2$ real roots if $m$ is even. Thus, for the usual case of $w(x)=1$, a $2 n+2$ point extension exists in the Gauss case if $n / 2$ is odd and in the Lobatto case if $n / 2$ is even. The formulas in the Lobatto case, corresponding to the Gauss formulas (70)-(74), are as follows:

Let $\hat{y}_{i}$ be the $n+1$ real zeros of $\tilde{E}_{n+1, \mu+1}$. Then

$$
\begin{aligned}
I f= & \sum_{i=1}^{n+1} \bar{r}_{i} f\left(\bar{x}_{i}\right)+\sum_{i=1}^{n+1} \bar{s}_{i} f\left(\hat{y}_{i}\right)+k_{n-1, \mu+1}^{-1} 2^{-n+1} \lambda_{n-1, \mu+1}^{-1} \\
& \times \int_{-1}^{1} w(x)\left(1-x^{2}\right) C_{n-1, \mu+1}(x) \tilde{E}_{n+1, \mu+1}(x) \\
& \quad \times f\left[\bar{x}_{1}, \ldots, \bar{x}_{n+1}, \hat{y}_{1}, \ldots, \hat{y}_{n+1}, x\right] d x,
\end{aligned}
$$

with $E P=3 n+2$, where

$$
\begin{gathered}
\bar{r}_{i}=\frac{\bar{x}_{i} \bar{u}_{i} E_{n, \mu+1}\left(\bar{x}_{i}\right)-\tilde{a}_{n-1, \mu+1} \bar{w}_{i}}{\bar{x}_{i} E_{n, \mu+1}\left(\bar{x}_{i}\right)-\tilde{a}_{n-1, \mu+1}}, \\
\bar{s}_{i}=\frac{\delta_{\mu+1}}{\left(1-\hat{y}_{i}^{2}\right) C_{n-1, \mu+1}\left(\hat{y}_{i}\right)\left(E_{n, \mu+1}\left(\hat{y}_{i}\right)+\hat{y}_{i} E_{n, \mu+1}^{\prime}\left(\hat{y}_{i}\right)\right)},
\end{gathered}
$$

and $\bar{x}_{i}, \bar{u}_{i}, \bar{w}_{i}$ are as in (8) and (10). From (19)-(22) with

$$
P_{N}(x)=\left(1-x^{2}\right) C_{n-1, \mu+1}(x) \tilde{E}_{n+1, \mu+1}(x),
$$

we have that

$$
\begin{array}{r}
I(f ; \lambda)=\sum_{i=1}^{n+1} \bar{r}_{i} \frac{f\left(\bar{x}_{i}\right)}{\bar{x}_{i}-\lambda}+\sum_{i=1}^{n+1} \bar{s}_{i} \frac{f\left(\hat{y}_{i}\right)}{\hat{y}_{i}-\lambda}+\bar{s}_{0} f(\lambda) \\
+k_{n-1, \mu+1}^{-1} 2^{-n+1} \lambda_{n-1, \mu+1}^{-1} \int_{-1}^{1} w(x)\left(1-x^{2}\right) C_{n-1, \mu+1}(x) \tilde{E}_{n+1, \mu+1}(x) \\
\times f\left[\bar{x}_{1}, \ldots, \bar{x}_{n+1}, \hat{y}_{1}, \ldots, \hat{y}_{n+1}, \lambda, x\right] d x,
\end{array}
$$

with $E P=3 n+3$, where

$$
\bar{s}_{0}=\frac{Q_{n-1, \mu+1}(\lambda)}{\bar{C}_{n+1, \mu+1}(\lambda)}-\frac{\lambda \delta_{\mu+1}}{\tilde{E}_{n+1, \mu+1}(\lambda) \bar{C}_{n+1, \mu+1}(\lambda)} .
$$

The same remarks about loss of significance apply here as in the Gauss case.

Department of Applied Mathematics

The Weizmann Institute of Science

Rehovot, Israel

1. P. J. Davis \& P. Rabinowitz, Methods of Numerical Integration, Academic Press, New York, 1975.

2. D. Elliot \& D. F. PAGET, "Gauss type quadrature rules for Cauchy principal value integrals," Math. Comp., v. 33, 1979, pp. 301-309.

3. W. Gautschi, "A survey of Gauss-Christoffel quadrature formulae," E. B. Christoffel. The Impact of His Work on Mathematics and the Physical Sciences (P. L. Butzer and F. Feher, Editors), Birkhäuser Verlag, Basel, 1981, pp. 72-147. 
4. (i. Monegato, "On polynomials orthogonal with respect to particular variable-signed weight functions," 7. Angew. Math. Phis., v. 31. 1980, pp. 549-555.

5. (3. MONEGATO. "Stieltjes polynomial and related quadrature rules," SIAM Rev.. v. 24. 1982. pp. $137-158$.

6. R. Piessens, E. de Doncker, C. Uberhuber \& D. Kahaner, QuadPaCK. A Quadranur' Subroutine Package. (To appear.)

7. R. Piessens, M. van Roy-Branders \& I. Mertens, "The automatic evaluation of Cauchy principal value integrals," Angew. Informatik, v. 18. 1976, pp. 31-35.

8. P. Rabinowitz. "The numerical evaluation of Cauchy principal value integrals," Fourth Sirmposium on Numerical Mathematics. Computer Science Dept.. Univ. of Natal, Durban. 1978. pp. 54-82.

9. P. RaBinowitz. "The exact degree of precision of generalized (jauss-Kronrod integration rules." Math. Comp., v. 35. 1980, pp. 1275-1283.

10. (j. SzEgö. "Üher gewisse orthogonale Polynome, die zu ciner oszillierenden Belegungsfunktion gehören." Math. Ann.. v. 110, 1934, pp. 501-513.

11. (i. Szegö. Orthogonal Pollnomials, 4th ed.. Amer. Math. Soc. Colloq. Publ.. Vol. 23. Amer. Math. Soc., Providence. R. I.. 1975: reprinted 1978. 